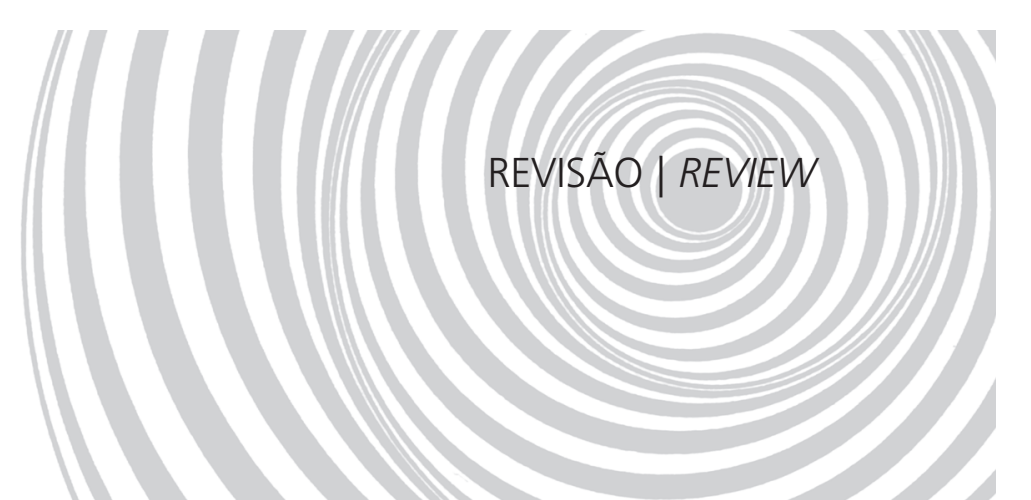

\title{
Desnutrição perinatal e o controle hipotalâmico do comportamento alimentar e do metabolismo do músculo esquelético
}

\author{
Perinatal undernutrition and hypothalamic \\ control of food intake and energy \\ metabolism in the skeletal muscle
}

\author{
Adriano BENTO-SANTOS ${ }^{1}$ \\ Leonardo dos Reis SILVEIRA ${ }^{2}$ \\ Raul MANHÃES-DE-CASTRO ${ }^{3}$ \\ Carol Gois LEANDRO 4
}

RE S U M O

\begin{abstract}
A deficiência de nutrientes durante os períodos críticos do desenvolvimento tem sido associada com maior risco para desenvolver obesidade e diabetes Mellitus na vida adulta. Um dos mecanismos propostos refere-se à regulação do comportamento alimentar e às alterações do metabolismo energético do músculo esquelético. Recentemente, tem sido proposta a existência de uma comunicação entre o hipotálamo e o músculo esquelético a partir de sinais autonômicos que podem explicar as repercussões da desnutrição perinatal. Assim, esta revisão tem como objetivo discutir as repercussões da desnutrição perinatal sobre o comportamento alimentar e o metabolismo energético muscular e a comunicação existente entre o hipotálamo e o músculo via sinais adrenérgicos. Foram utilizadas as bases de dados MedLine/PubMed, Lilacs e Bireme, com publicações entre 2000 e 2011. Os termos de indexação utilizados foram: feeding behavior, energy metabolism, protein malnutrition, developmental plasticity, skeletal muscle e autonomic nervous system. Concluiu-se que a desnutrição perinatal pode atuar no controle hipotalâmico do comportamento alimentar e no metabolismo energético muscular, e a comunicação hipotálamo-músculo pode favorecer o desenvolvimento de obesidade e comorbidades durante o desenvolvimento.
\end{abstract}

Termos de indexação: Comportamento alimentar. Desnutrição. Doenças metabólicas. Hiperfagia. Hipotálamo. Plasticidade fenotípica, desenvolvimento.

\footnotetext{
1 Universidade Federal de Pernambuco, Centro de Ciências da Saúde, Programa de Pós-Graduação em Neuropsiquiatria e Ciências do Comportamento. Av. Prof. Moraes Rego, s/n., Cidade Universitária, 50670-901, Recife, PE, Brasil. Correspondência para/Correspondence to: A BENTO-SANTOS. E-mail: <absbio@yahoo.com.br>.

2 Universidade de São Paulo, Escola de Educação Física e Esporte. Ribeirão Preto, SP, Brasil.

3 Universidade Federal de Pernambuco, Centro de Ciências da Saúde, Departamento de Nutrição. Recife, PE, Brasil.

${ }^{4}$ Universidade Federal de Pernambuco, Centro Acadêmico de Vitória, Núcleo de Educação Física e Ciências do Esporte. Vitória de Santo Antão, PE, Brasil.
} 
404 | A BENTO-SANTOS et al.

\section{A B S T R A C T}

Undernutrition during the critical period of development has been associated with susceptibility to obesity and diabetes Mellitus in adulthood. One of the underlying mechanisms can be related with the relationship between the food intake and the metabolism of skeletal muscle. A communication between the hypothalamus and skeletal muscle has been recently proposed, which can explain the repercussion of perinatal undernutrition. Thus, this review aims mainly to discuss the repercussions of perinatal undernutrition on food intake and skeletal muscle metabolism by adrenergic signals. Articles published from 2000 to 2011 were searched in the Medline/Pubmed, Lilacs and Bireme databases using the following keywords: feeding behavior, energy metabolism, protein malnutrition, developmental plasticity, skeletal muscle and autonomic nervous system. In conclusion, perinatal undernutrition can alter the hypothalamic control of food intake and skeletal muscle metabolism. Additionally, communication between the hypothalamus and skeletal muscle can promote the development of obesity and associated diseases.

Indexing terms: Feeding behavior. Malnutrition. Metabolic diseases. Hyperphagia. Hypothalamus. Phenotypic plasticity, growth.

\section{N T R O D U ÇÃ O}

O crescimento e o desenvolvimento do indivíduo são marcados por períodos de rápida proliferação e diferenciação celular (tal como gestação, lactação e primeira infância) ${ }^{1}$. Esses períodos são críticos para o desenvolvimento uma vez que órgãos e sistemas são vulneráveis às alterações ambientais ${ }^{1,2}$. Uma possível explicação para a relação entre agressões sofridas nos períodos críticos e as repercussões tardias sobre os sistemas fisiológicos é proposta pela plasticidade fenotípica, que corresponde à capacidade de o indivíduo modificar o fenótipo de acordo com alterações ambientais ${ }^{3}$. A desnutrição perinatal é um dos fatores ambientais mais bem estudados como indutor de plasticidade fenotípica ${ }^{4,5}$. Estudos com animais e humanos têm demonstrado que a desnutrição quando aplicada nos períodos críticos aumenta a predisposição do indivíduo em desenvolver doenças cardiovasculares ${ }^{6,7}$, fatores de risco associados com a hipertensão arterial, resistência à insulina ${ }^{8}$, hiperlipidemia ${ }^{5}$ e obesidade na vida adulta ${ }^{4}$.

Estudos recentes têm demonstrado que uma dieta pobre em proteína ( $8 \%$ de caseína) no período de gestação e lactação leva a alterações permanentes na estrutura e na funcionalidade do sistema nervoso central9,10. Essas alterações podem ser de ordem química e/ou morfológica e ocorrer em diversas estruturas, como o hipotálamo, que tem papel chave no controle da expressão comportamental e da homeostase energética ${ }^{11}$. O hipotálamo é o centro regulador do comportamento alimentar e pode atuar no metabolismo energético no músculo e ambos podem apresentar mudanças fenotípicas induzidas pela desnutrição perinatal ${ }^{12,13}$. A desnutrição perinatal induz hiperfagia na prole, promove alterações na expressão de neurotransmissores orexigênicos e anorexigênicos, na expressão de genes envolvidos com a regulação de sensores de nutrientes hipotalâmicos e no controle do metabolismo ${ }^{14,15}$. Da mesma forma, estudos experimentais demonstram que filhotes de ratas expostas à desnutrição proteica durante a gestação e lactação apresentam alteração na quantidade e proporções de fibras musculares e resistência à insulina na idade adulta $8,16,17$.

O presente estudo teve como objetivo discutir os danos induzidos pela desnutrição no início da vida sobre o controle hipotalâmico do comportamento alimentar e no metabolismo energético no músculo. Ademais, abordamos a comunicação existente entre o hipotálamo e o músculo esquelético via alterações nos sensores nutricionais hipotalâmicos e nos receptores adrenérgicos no músculo.

\section{MÉTODOS}

Para realização desta revisão, utilizou-se como critério de inclusão a seleção de artigos publicados nas bases de dados MedLine/PubMed, Lilacs e Bireme, que tinham como termos de indexação: feeding behavior, energy metabolism, protein undernutrition, developmental plasticity, 
skeletal muscle e autonomic nervous system. Dentre os artigos selecionados, estão inclusos estudos clássicos sobre "programação fetal" a partir de 1964 e estudos atuais sobre plasticidade fenotípica. Para discussão sobre nutrição, comportamento alimentar e metabolismo energético, foram utilizados artigos publicados entre os anos de 2000 e 2011. Este estudo foi realizado nos meses de abril e março de 2011.

\section{Papel do hipotálamo no controle do comportamento alimentar e do metabolismo energético no músculo esquelético}

O hipotálamo, dentre suas inúmeras funções, tem papel de centro regulador do comportamento alimentar ${ }^{13}$. O comportamento alimentar refere à interação de diversos processos que envolvem a escolha do alimento, início e término da alimentação, a frequência, o tempo e o tamanho da refeição ${ }^{18}$. A regulação do comportamento alimentar ocorre por meio da complexa interação entre mecanismos periféricos e centrais que controlam a fome e a saciedade ${ }^{19}$. O controle central do comportamento alimentar é feito pelo hipotálamo que monitora, processa e gera respostas aos sinais de nutrientes, sinais hormonais periféricos e sinais neurais, que refletem mudanças nos níveis de energia dos animais superiores ${ }^{20-22}$. Esse controle é feito por ações coordenadas entre os diversos núcleos hipotalâmicos, entre eles o Núcleo Ventromedial (NVM), o Núcleo Paraventricular (NPV) e o Núcleo Arqueado (ARQ).

O Núcleo Arqueado tem papel de destaque no con-trole do comportamento alimentar e é um dos núcleos hipotalâmicos mais estudados ${ }^{21-}$ ${ }^{23}$. Nele, há populações de neurônios que expressam neuropeptídeos que estimulam a fome (orexigênicos) e neuropeptídeos que geram saciedade (anorexigênicos) 21,22. Os neurônios orexigênicos coexpressam o Neuropeptídeo Y (NPY) e o Peptídeo Relacionado ao Gene Agouti (AgRP), enquanto os neurônios anorexigênicos coexpres-sam o transcrito regulado por Cocaína e Anfetamina (CART) e o Hormônio Pro-Opiomelanocortina (POMC) $)^{21,22,24}$. O POMC dá origem ao Hormônio Estimulador de Melanócitos alfa $(\alpha-M S H)$, que exerce papel-chave sobre a inibição da ingestão alimentar ${ }^{20}$. A ação desses neuropeptídeos hipotalâmicos é que determina a busca e a ingestão de alimentos ou o término da alimentação.

A expressão dos neuropeptídeos orexigênicos e anorexigênicos é modulada por sinais hormonais periféricos e pelo estado energético do indivíduo. Os hormônios insulina e leptina estão presentes em altas concentrações plasmáticas após as refeições, e, com o aumento de adiposidade, eles atuam no núcleo arqueado, inibindo a expressão de NPY e AgRP e, simultaneamente, estimulando a expressão de $\alpha-M S H$ a partir do POMC. Isso gera redução da ingestão alimentar e aumento do dispêndio energético ${ }^{20}$. Há neurônios hipotalâmicos que agem como sensores de nutrientes, devido a sua capacidade de responder às mudanças na disponibilidade de nutrientes circulantes (como glicose e lipídeos) e às mudanças na concentração de moléculas intracelulares, como os Ácidos Graxos de Cadeia Longa Ativados (LCFA - CoA) e a malonil CoA (um precursor da síntese de ácidos graxos) ${ }^{25-27}$.

Alguns estudos indicam que neurônios POMC, NPY e AgRP são sensíveis a mudanças na disponibilidade de nutrientes ${ }^{27-31}$. Em resposta a um aumento nos níveis de nutrientes, neurônios POMC despolarizam enquanto neurônios NPY e AgRP hiperpolarizam, gerando, respectivamente, aumento e diminuição da liberação de seus neurotransmissores ${ }^{27-31}$. Outros estudos demonstram que durante o jejum há uma redução na concentração hipotalâmica de LCFA - CoA, e de malonil CoA, levando a uma diminuição na expressão de POMC, bem como a um aumento na expressão de NPY e AgRP, que induz a ingestão de alimentos e o ganho de peso ${ }^{23}$. Ademais, o uso da droga $\mathrm{C} 75$, que promove aumento na concentração de malonil CoA hipotalâmica, induziu em camundongos: bloqueio da ingestão alimentar por inibição da expressão de NPY e AgRP; aumento da expressão de POMC e perda de peso ${ }^{23,32,33}$. 
406 | A BENTO-SANTOS et al.

Dessa forma, alterações na quantidade e na atividade de neurônios que expressam esses neuropeptídeos podem levar a mudanças no comportamento alimentar e na homeostase energética.

\section{Plasticidade fenotípica, desnutrição perinatal e controle hipotalâmico do comportamento alimentar}

O termo "plasticidade fenotípica" refere-se à capacidade de mudança do fenótipo em resposta às alterações ambientais durante períodos críticos do desenvolvimento e às repercussões na vida adulta ${ }^{3}$. A hipótese da plasticidade fenotípica, inicialmente chamada de "programação" 34 , propõe que o risco de desenvolver uma doença é consequência da transição de um ambiente uterino adverso para um ambiente pós-natal enriquecido ${ }^{35}$. A desnutrição perinatal é um dos fatores ambientais mais bem estudados como indutor de plasticidade fenotípica ${ }^{4,5}$. Estudos com humanos têm demonstrado que a desnutrição perinatal aumenta a predisposição do indivíduo a desenvolver doenças cardiovasculares ${ }^{6,7}$ e fatores de risco associados, como hipertensão arterial, resistência à insulina, hiperlipidemia ${ }^{5}$ e obesidade ${ }^{4}$. Em ratos, foi demonstrado que restrição alimentar (30\% - 50\% da ingestão do controle) ou dieta hipoprotéica ( $8 \%$ de caseína) no período neonatal parecem provocar deficits no desenvolvimento do cérebro e dos rins ${ }^{36}$, além de alterações morfológicas e funcionais no coração e no músculo esquelético ${ }^{12,37,38}$.

Estudos recentes têm demonstrado que uma dieta pobre em proteína ( $8 \%$ de caseína) no período de gestação e lactação induz atraso na ontogênese dos reflexos e deficits no desenvolvimento de padrões motores e comportamentais de ratos $^{9,10}$. Outros estudos mostram que dieta com aporte inadequado de nutrientes no período de lactação induz efeitos permanentes sobre o tamanho do encéfalo, número de neurônios, aprendizagem e memória ${ }^{39,40}$. De fato, regiões cerebrais que controlam determinados comportamentos podem ser irreversivelmente modifi- cadas pela desnutrição no período crítico. Essas alterações podem ser de ordem química e/ou morfológica e ocorrer em diversas estruturas, como o hipotálamo, que tem papel chave no controle da expressão comportamental e da homeostase energética ${ }^{11}$.

Estudos com ratos demonstraram que restrição alimentar (30\% da ingestão do controle) e desnutrição proteica ( $8 \%$ de caseína) durante a gestação e lactação induzem hiperfagia e preferência por alimentos calóricos na prole ao longo do desenvolvimento até a idade adulta ${ }^{41,42}$. Esses efeitos parecem estar associados a um atraso na sensação de saciedade, um aumento no tamanho da refeição e uma redução no período de latência para comer ${ }^{41,42}$. Os mecanismos associados parecem ter relação com a redução na ação anoréxica da insulina, leptina e serotonina e de neuropeptídeos que controlam o comportamento alimen$\operatorname{tar}^{43}$. De fato, ratos submetidos à desnutrição proteica (caseína 8\%) na gestação e lactação apresentaram hiperfagia após o desmame, aos 35 dias e aos 60 dias de vida, e uma evidente redução da ação inibitória da serotonina na ingestão alimentar via diminuição da sensibilidade dos receptores $5 \mathrm{HT}_{1 \mathrm{~b}}{ }^{43}$. É interessante observar que ratos jovens que foram desnutridos precocemente apresentam uma maior expressão hipotalâmica de NPY e AgRP e uma menor expressão de POMC ${ }^{15}$. Contudo, a partir dos 90 dias de vida, não foi observada hiperfagia nos animais, que, mesmo assim, desenvolveram, na vida adulta, um fenótipo de obesidade com aumento da gordura abdominal e dos níveis séricos de triglicerídeos e ácidos graxos ${ }^{15}$. Isso sugere que a programação da obesidade e de doenças correlatas pode ter mecanismos independentes dos efeitos da desnutrição sobre o comportamento alimentar e estar relacionada ao metabolismo no músculo esquelético.

\section{Desnutrição perinatal e metabolismo no músculo esquelético}

O músculo esquelético, que tem um papel fundamental no metabolismo dos carboidratos, 
lipídeos e proteínas, também tem sido alvo de estudos que associam a desnutrição perinatal e as doenças metabólicas da vida adulta ${ }^{12,17,44}$. Estudos epidemiológicos demonstram que as mudanças no fenótipo da composição corporal estão associadas ao aumento do percentual de gordura corporal e à redução na massa muscular na vida adulta ${ }^{45}$. Tem sido especulado que alterações no crescimento e desenvolvimento de fibras musculares podem ser um dos mecanismos subjacentes à programação de doenças metabólicas ${ }^{46}$. Em modelos animais têm sido demonstrado que a desnutrição durante a gestação parece reduzir a taxa de crescimento e o número de fibras musculares de ratos na vida adulta ${ }^{47}$. Ratos jovens ( 4 semanas de vida) que sofreram desnutrição proteica ( $9 \%$ de caseína) durante a gestação apresentaram redução no número e na densidade de fibras musculares ${ }^{48}$. Bayol et al. ${ }^{44}$ encontraram uma redução no número de núcleos dos músculos semitendinoso e gastrocnêmio, o que indica uma diminuição na proliferação celular em ratos jovens que sofreram restrição de dieta no período de gestação ( $40 \%$ da dieta consumida pelos controles).

Alterações sobre o tecido muscular parecem atuar como um fator etiológico da resistência à insulina e de doenças como obesidade e diabetes Mellitus ${ }^{8,16,47}$. Estudos experimentais demonstram que filhotes de ratas expostas à desnutrição proteica ( $8 \%$ de caseína) durante a gestação e lactação apresentam uma diminuição no percentual de fibras oxidativas (Tipo I) e aumento no percentual de fibras glicolíticas (Tipo lla e llb) ${ }^{49}$. Essas alterações podem estar relacionadas ao aparecimento precoce da resistência à insulina na idade adulta. Ratos adultos submetidos à desnutrição perinatal apresentam resistência à insulina associada a uma redução na expressão dos Transportadores de Glicose (GLUT-4), proteínas p85 e p110 3 (subunidades da fosfatidilinositol 3-kinase) e de Proteína Kinase $C$ zeta $(\mathrm{PKC} \zeta)^{8,16}$. Essas proteínas estão envolvidas positivamente com a propagação do sinal intracelular da insulina, com a translocação do GLUT-4 e com o transporte de glicose através da membrana sarcoplasmática ${ }^{8,16}$.
Um dos mecanismos propostos para o aparecimento precoce da resistência periférica à insulina pode estar associado à concentração elevada de Triglicerídeos Plasmáticos e Intramusculares (TGIM) ${ }^{50,51}$. O aumento de TGIM eleva o conteúdo LCFA-CoA e Diacilglicerol (DAG) ${ }^{52}$, este último atua inibindo a propagação do sinal intracelular da insulina ${ }^{51,53}$. O DAG estimula a Proteína Kinase $C$ teta $(P K C \theta)$ a inativar o Substrato Receptor de Insulina 1 (IRS-1) através de fosforilação do seu resíduo serina ${ }^{51,53}$. Em uma prole exposta à desnutrição intrauterina, foi observada uma maior concentração sérica de ácidos graxos livres, além da menor expressão de enzimas-chave da via de sinalização da insulina aos 120 dias $^{54}$. Ratos adultos cujas mães foram submetidas à restrição alimentar $(70 \%$ da dieta ofertada ao controle) apresentaram maior gordura suprarrenal relativa ao peso corporal, maior concentração sérica de leptina, insulina e glicose, considerados parte dos indicadores do fenótipo da obesidade ${ }^{55}$. Dessa forma, alterações na expressão de proteínas da cascata de sinalização da insulina, bem como aumento nos níveis de lipídeos, como observado em animais desnutridos no período perinatal ${ }^{14,15}$, comprometem a tolerância e a captação de glicose no músculo esquelético.

Além dos efeitos diretos no músculo sobre a morfologia e a sensibilidade à insulina, é possível que a desnutrição perinatal modifique o metabolismo muscular através da alteração do controle hipotalâmico via Sistema Nervoso Autônomo (SNA).

\section{Comunicação entre o hipotálamo e o músculo esquelético e a desnutrição perinatal}

Recentemente, tem sido reconhecido o papel do hipotálamo na homeostase energética através do controle do metabolismo. Por meio do SNA, o hipotálamo se comunica e modula o metabolismo energético em órgãos periféricos como o fígado, o tecido adiposo e o músculo esquelético ${ }^{20-22,32,56,57}$. Do hipotálamo, partem neurônios POMC que fazem sinapses com neurônios pré-ganglionares, localizados na coluna médio-lateral 
408 | A BENTO-SANTOS et al.

da medula espinal, e estes se comunicam com o músculo esquelético por meio de fibras pósganglionares simpáticas ${ }^{56,59}$.

O hipotálamo emite sinais que geram mudanças no metabolismo energético do músculo esquelético ${ }^{32}$. O sinal conduzido por meio do sistema nervoso simpático é transmitido ao músculo através da ligação de catecolaminas (noradrenalina ou adrenalina) aos receptores adrenérgicos que, acoplados à proteína $G$, estimulam a adenilato ciclase a aumentar os níveis de Adenosina 3' 5' Monofosfato (AMP) e AMP cíclico (AMP-c) $)^{32,57 .}$
O aumento de AMP-c ativa a Proteína Quinase A (PKA), estimulando, dessa forma, a Lípase Sensível a Hormônios (LSH) a quebrar triglicerídeos em ácidos graxos e glicerol, aumentando a disponibilidade de ácidos graxos para serem oxidados na mitocôndria. O AMP ativa a proteína quinase dependente de AMP (AMPK), que catalisa a inativação da enzima acetil CoA Carboxilase (ACC), levando a uma diminuição nos níveis de malonil CoA no músculo, um potente inibidor alostérico da Carnitina Acil Transferase 1 (CAT-1) (Figura 1). A diminuição de malonil CoA cessa o efeito

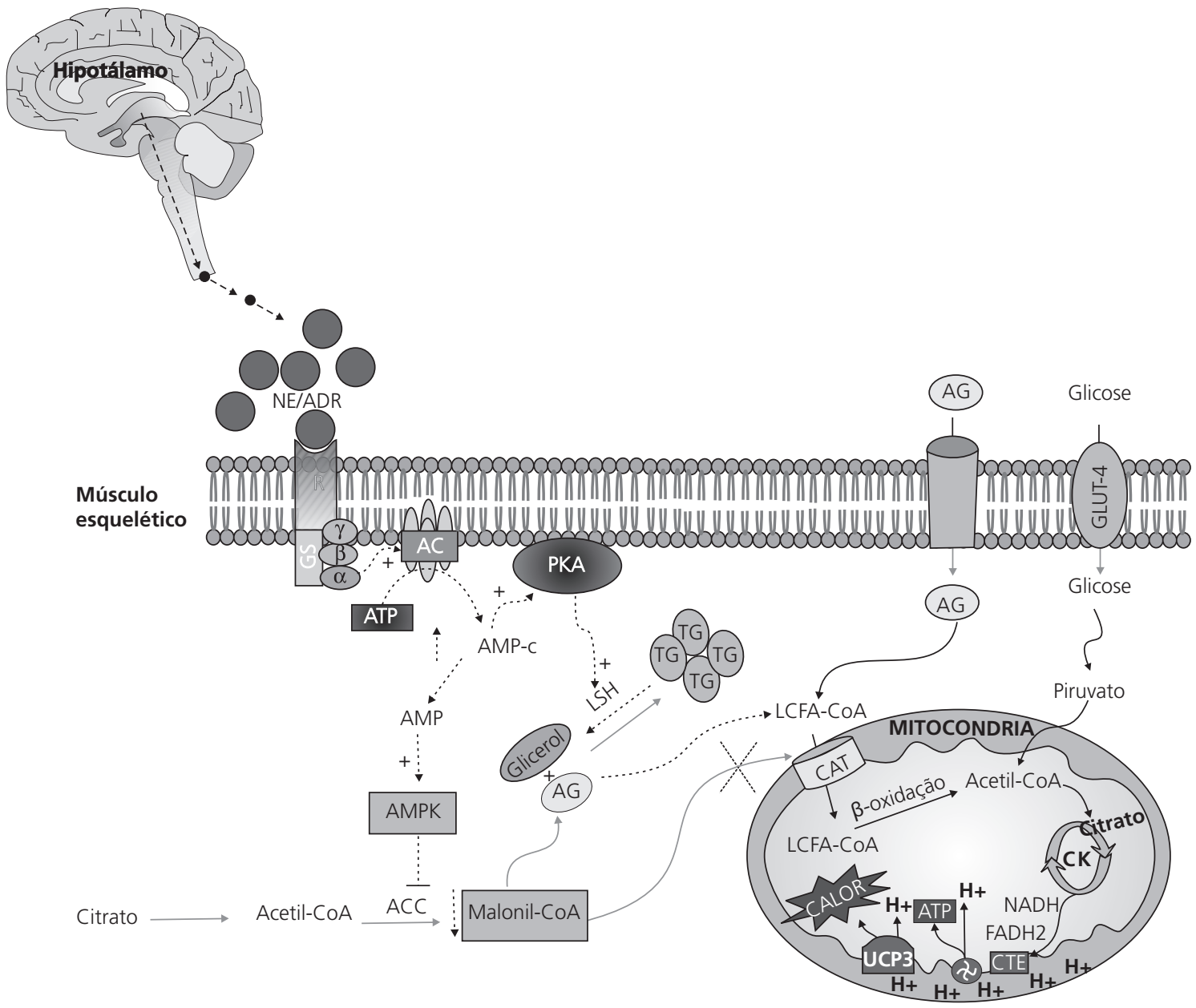

Figura 1. Modulação do metabolismo energético muscular por meio de sinais adrenérgicos. As setas contínuas (cinzas) representam os efeitos induzidos pelo aumento da concentração de citrato, em decorrência do estado energético positivo, enquanto as setas tracejadas representam os efeitos intracelulares no músculo esquelético gerados pela propagação do sinal adrenérgico.

Notas: AG: Ácidos Graxos; LCFA-CoA: Ácidos Graxos de Cadeia Longa Ativados; CAT: Complexo Carnitina Acil-Transferase; CK: Ciclo de Krebs; CTE: Cadeia Transportadora de Elétrons; UCP-3: Proteína Desacopladora; GLUT-4: Transportador de Glicose; NE: Noradrenalina; ADR: Adrenalina; R: Receptor Adrenérgico; G-s: Proteína G Estimulatória; AC: Adenilato Ciclase; ATP: Trifosfato de Adenosina; AMP-C: AMP Cíclico; AMP: Adenosina 3' 5' Monofosfato; AMPK, Proteína Kinase Dependente de AMP; ACC: Acetil; CoA Carboxilase; PKA: Proteína Kinase A; LSH: Lípase Sensível a Hormônio; TG: Triglicerídeos. 
inibitório sobre a CAT-1, facilitando a entrada de LCFA-CoA na mitocôndria para ser oxidado através da $\beta$ - oxidação ${ }^{32,57}$. Outra resposta metabólica muscular ao sinal hipotalâmico é a elevação da termogênese, através de aumento na expressão de proteínas Desacopladoras Mitocondriais no músculo (UCP-3) $)^{32,57}$. A UCP-3 dissipa o gradiente de prótons do espaço intermembranar mitocondrial, e produz calor ao invés de produzir ATP, aumentando assim o gasto energético ${ }^{60}$.

Os danos induzidos pela desnutrição no início da vida sobre o controle hipotalâmico do metabolismo energético no músculo podem ser originados por alterações nos sensores nutricionais hipotalâmicos e na transmissão do sinal simpático via receptores adrenérgicos no músculo (Figura 2). Ratos adultos (180 dias) provindos de mães desnutridas (8\% caseína) na gestação e lactação apresentam alterações na expressão de genes em áreas do hipotálamo que respondem à sinalização da insulina e à presença de nutrientes, particularmente de lipídeos ${ }^{61}$. Da mesma forma, a desnutrição perinatal alterou a expressão de genes que regulam o metabolismo dos lipídeos e dos carboidratos no hipotálamo, tais como os receptores de glicocorticoides, Receptor Betatireoidiano (TRb), o receptor gama ativado pelo Proliferador de Peroxissoma (PPAR-gama), e o Receptor alfa $X$ Retinoide (RXRa) ${ }^{61}$.

Estudos experimentais apontam os efeitos de sinais hipotalâmicos sobre o metabolismo energético no músculo esquelético ${ }^{32,57}$. Camundongos foram submetidos ao jejum ou ao jejum mais administração da droga C75, um inibidor da síntese de ácidos graxos no hipotálamo ${ }^{32,57}$. Animais que receberam $\mathrm{C} 75$ apresentaram maiores concentrações de malonil CoA hipotalâmica e dispêndio energético elevado no músculo ${ }^{32,57}$. Esses animais apresentaram diminuição da concentração de malonil CoA muscular e aumento na expressão de UCP-3, o que resultou numa maior taxa de oxidação de ácidos graxos ${ }^{32,57}$. Esses resultados não foram observados com a utilização de bloqueadores adrenérgicos, o que sugere um importante e potente controle do metabolismo muscular pelo hipotálamo através do SNA ${ }^{32,57}$.
O sistema simpato-adrenérgico é alterado permanentemente em resposta ao estresse materno, administração de glicocorticoides ou ingestão materna de uma dieta baixa em proteína ${ }^{62}$. A concentração de catecolaminas circulantes e a expressão de adrenorreceptores no tecido adiposo subcutâneo e intra-abdominal são alteradas em filhotes ( 12 semanas de idade) submetidos a uma dieta materna baixa em proteína ${ }^{63}$. Da mesma forma, a restrição proteica na gestação diminui a responsividade de receptores $\beta$-adrenérgicos e da insulina no músculo cardíaco, predispondo os filhotes (3 meses de idade) ao risco de deficit cardiovascular e falha cardíaca ${ }^{64}$. No músculo esquelético, as catecolaminas estimulam a oxidação de glicose e ácidos graxos e tem um efeito importante no metabolismo proteico ${ }^{65}$. As análises histoquímicas sugerem que esse efeito seja músculo-específico, sendo mais evidenciado em músculo com predominância de contração rápida e perfil metabólico glicolítico ${ }^{66}$. Outros estudos demonstraram que alterações no SNA e na atividade de enzimas envolvidas no metabolismo lipídico têm sido associadas a doenças como diabetes Mellitus tipo II e obesidade, através de influência sobre o gasto energético 67,68 .

O controle do metabolismo energético por sinais adrenérgicos parece ter importante papel na regulação do dispêndio energético e pode estar envolvido no surgimento de doenças metabólicas. Assim, animais desnutridos no período perinatal podem apresentar redução do metabolismo energético muscular em decorrência de alterações no controle hipotalâmico, que resultam em menor densidade e/ou menor responsividade de receptores adrenérgicos, menor expressão de UCP-3 e menor atividade de enzimas envolvidas na oxidação de lipídeos, além de apresentar aumento da esterificação de ácidos graxos com acúmulo de triglicerídeos. Isso poderia explicar os resultados encontrados em ratos submetidos à desnutrição perinatal que não apresentaram hiperfagia na vida adulta, contudo desenvolveram obesidade e apresentaram aumento da gordura abdominal, dos níveis de triglicerídeos e ácidos graxos plasmáticos ${ }^{14,15}$. 
410 | A BENTO-SANTOS et al.

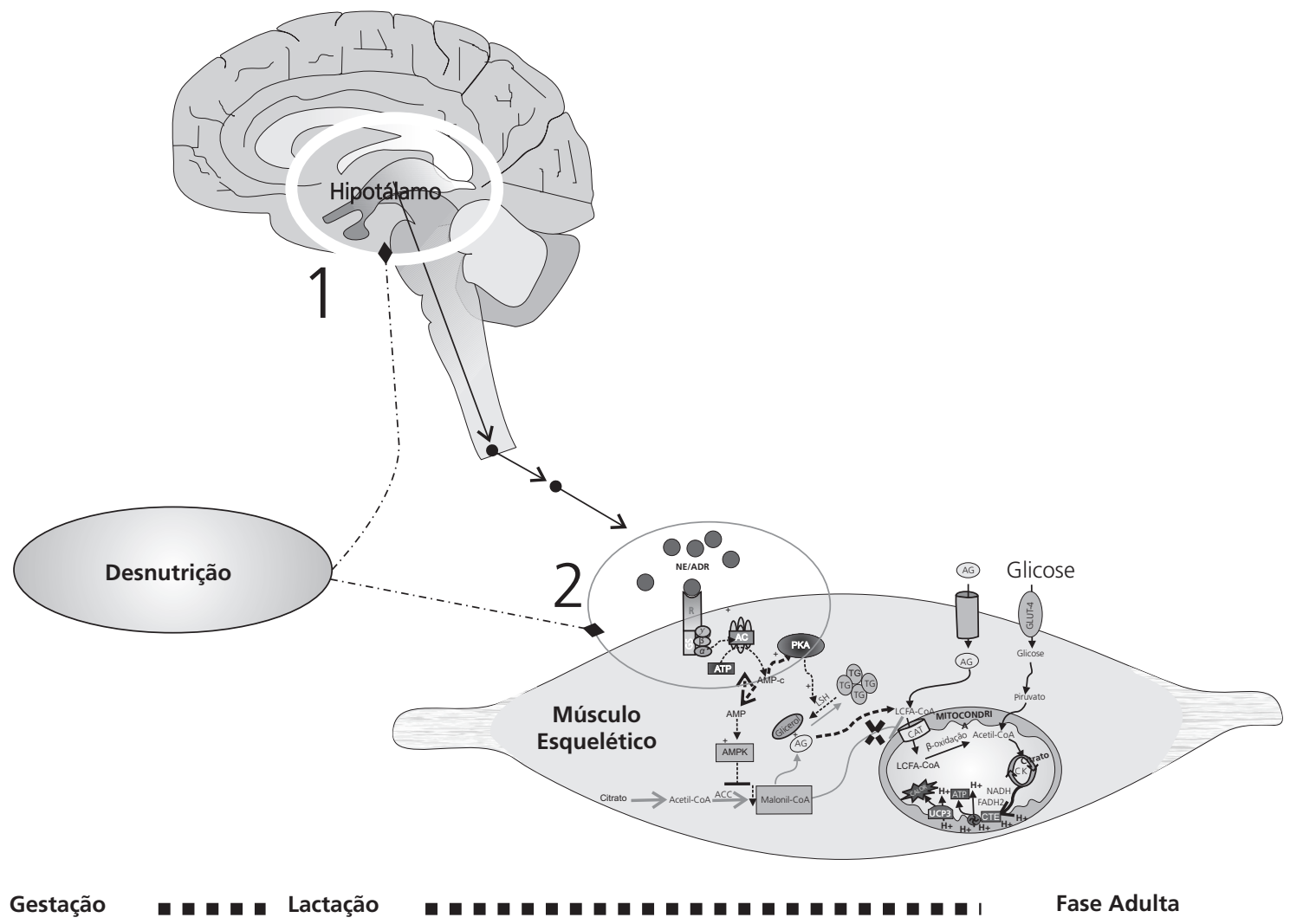

Plasticidade do organismo

Figura 2. Desnutrição nos períodos de alta plasticidade do organismo pode induzir alterações no hipotálamo e no músculo esquelético. Na idade adulta, estas alterações podem repercutir no controle hipotalâmico do metabolismo muscular via sistema adrenérgico. Dentre os locais cuja desnutrição perinatal parece induzir alterações estão: 1 - sensores de nutrientes hipotalâmicos; 2 - ligação e sinalização das catecolaminas nos receptores adrenérgicos musculares.

\section{O N CLUSÃ O}

Em resposta a estímulos ou insultos ambientais, a plasticidade fenotípica possibilita a ocorrência de mudanças comportamentais, estruturais e metabólicas no indivíduo. A desnutrição no início da vida induz alterações no comportamento alimentar em direção à hiperfagia e à preferência por alimentos calóricos. No músculo esquelético, a desnutrição perinatal parece alterar o fenótipo de fibras e causar resistência periférica à insulina. Tendo em vista que os nutrientes podem modular a expressão de neuropeptídios e sinais hipotalâmicos que modulam o comporta- mento alimentar e o metabolismo energético periférico, é possível que alterações induzidas pela desnutrição perinatal no metabolismo muscular sejam originadas por falha no controle do metabolismo energético pelo hipotálamo.

Dessa forma, estudos que relacionem a programação do controle hipotalâmico sobre o metabolismo energético muscular através da comunicação hipotálamo-músculo são necessários para o entendimento dos mecanismos subjacentes ao surgimento precoce de obesidade e comorbidades associadas à desnutrição no início da vida. 


\section{COLABORADORES}

A BENTO-SANTOS participou da consulta as bases de dados e da escrita do artigo. LR SILVEIRA e $R$ MANHÃES-DE-CASTRO fizeram a revisão final do artigo. CG LEANDRO participou da escrita e revisão do artigo.

\section{REFERÊ N CIAS}

1. Winick M, Rosso P, Brasel JA. Malnutrition and cellular growth in the brain. Bibl Nutr Dieta. 1972; (17):60-8.

2. Dobbing J. The influence of early nutrition on the development and myelination of the brain. Proc $\mathrm{R}$ Soc Lond B Biol Sci. 1964;159(18):503-9. doi: 10.1098/rspb.1964.0016.

3. Gluckman PD, Hanson MA, Pinal C. The developmental origins of adult disease. Matern Child Nutr. 2005; 1(3):130-41. doi: 10.1111/j.17 40-8709.2005.00020.x.

4. Ravelli GP, Stein ZA, Susser MW. Obesity in young men after famine exposure in utero and early infancy. N Engl J Med. 1976; 295(7):349-53. doi: 10.1056/NEJM197608122950701.

5. Barker DJ. Fetal origins of cardiovascular disease. Ann Med. 1999; 31(Suppl 1):3-6.

6. Hales CN, Barker DJ. Type 2 (non-insulindependent) diabetes mellitus: the thrifty phenotype hypothesis. Diabetologia. 1992; 35(7):595-601.

7. Forsdahl A. Are poor living conditions in childhood and adolescence an important risk factor for arteriosclerotic heart disease? Br J Prev Soc Med. 1977; 31(2):91-5.

8. Ozanne SE, Jensen CB, Tingey KJ, Storgaard $H$, Madsbad S, Vaag AA. Low birthweight is associated with specific changes in muscle insulin-signalling protein expression. Diabetologia. 2005; 48(3): 547-52. doi: 10.1007/s00125-005-1669-7.

9. Barreto Medeiros JM, Cabral Filho JE, De Souza SL, Freitas Silva SR, Mendes Da Silva C, Deiro TC, et al. Early malnourished rats are not affected by anorexia induced by a selective serotonin reuptake inhibitor in adult life. Nutr Neurosci. 2002; 5(3): 211-4.

10. Falcao-Tebas F, Bento-Santos A, Fidalgo MA, de Almeida MB, dos Santos JA, Lopes de Souza S, et al. Maternal low-protein diet-induced delayed reflex ontogeny is attenuated by moderate physical training during gestation in rats. Br J Nutr. 2012; 107(3):372-7. doi: 10.1017/S0007114511002947.

11. Wauben IP, Wainwright PE. The influence of neonatal nutrition on behavioral development: a critical appraisal. Nutr Rev. 1999; 57(2):35-44.
12. Wilson SJ, Ross JJ, Harris AJ. A critical period for formation of secondary myotubes defined by prenatal undernourishment in rats. Development. 1988; 102(4):815-21.

13. Plagemann A, Waas $T$, Harder T, Rittel F, Ziska T, Rohde W. Hypothalamic neuropeptide $Y$ levels in weaning offspring of low-protein malnourished mother rats. Neuropeptides. 2000; 34(1):1-6. doi: 10.1054/npep.1999.0778.

14. Orozco-Solis R, Matos RJ, Lopes de Souza S, Grit I, Kaeffer B, Manhaes de Castro R, et al. Perinatal nutrient restriction induces long-lasting alterations in the circadian expression pattern of genes regulating food intake and energy metabolism. Int J Obes (Lond). 2011; 35(7):990-1000. doi: 10.10 38/ijo.2010.223.

15. Orozco-Solis R, Lopes de Souza S, Barbosa Matos RJ, Grit I, Le Bloch J, Nguyen P, et al. Perinatal undernutrition-induced obesity is independent of the developmental programming of feeding. Physiol Behav. 2009; 96(3):481-92. doi: 10.1016/ j.physbeh.2008.11.016.

16. Ozanne SE, Olsen GS, Hansen LL, Tingey KJ, Nave $\mathrm{BT}$, Wang $\mathrm{CL}$, et al. Early growth restriction leads to down regulation of protein kinase $C$ zeta and insulin resistance in skeletal muscle. J Endocrinol. 2003; 177(2):235-41. doi: 10.1677/joe.0.17702 35.

17. Toscano AE, Manhaes-de-Castro R, Canon F. Effect of a low-protein diet during pregnancy on skeletal muscle mechanical properties of offspring rats. Nutrition. 2008; 24(3):270-8. doi: 10.1016/j.nut.20 07.12 .004

18. Blundell J. Pharmacological approaches to appetite suppression. Trends Pharmacol Sci. 1991; 12(4): 147-57.

19. Nagase $H$, Nakajima A, Sekihara $H$, York DA, Bray GA. Regulation of feeding behavior, gastric emptying, and sympathetic nerve activity to interscapular brown adipose tissue by galanin and enterostatin: the involvement of vagal-central nervous system interactions. J Gastroenterol. 2002; 37(Suppl 14):118-27.

20. Schwartz MW, Woods SC, Porte D Jr, Seeley RJ, Baskin DG. Central nervous system control of food intake. Nature. 2000; 404(6778):661-71. doi: 10.1038/35007534.

21. Williams G, Bing C, Cai XJ, Harrold JA, King PJ, Liu $\mathrm{XH}$. The hypothalamus and the control of energy homeostasis: different circuits, different purposes. Physiol Behav. 2001; 74(4-5):683-701. doi: 5003 1938401006126.

22. Mercer JG, Speakman JR. Hypothalamic neuropeptide mechanisms for regulating energy balance: from rodent models to human obesity. Neurosci Biobehav Rev. 2001; 25(2):101-16. doi: S0 149-7634(00)00053-1. 
23. Shimokawa T, Kumar MV, Lane MD. Effect of a fatty acid synthase inhibitor on food intake and expression of hypothalamic neuropeptides. Proc Natl Acad Sci USA. 2002; 99(1):66-71. doi: 10.10 73/pnas.012606199.

24. Morton GJ, Cummings DE, Baskin DG, Barsh GS, Schwartz MW. Central nervous system control of food intake and body weight. Nature. 2006; 443(7109):289-95. doi: 10.1038/nature05026.

25. He W, Lam TK, Obici S, Rossetti L. Molecular disruption of hypothalamic nutrient sensing induces obesity. Nat Neurosci. 2006; 9(2):227-33. doi: 10.10 38/nn1626.

26. Hu Z, Cha SH, Chohnan S, Lane MD. Hypothalamic malonyl-COA as a mediator of feeding behavior. Proc Natl Acad Sci USA. 2003; 100(22):12624-9. doi: 10.1073/pnas.1834402100.

27. Ibrahim N, Bosch MA, Smart JL, Qiu J, Rubinstein M, Ronnekleiv OK, et al. Hypothalamic proopiomelanocortin neurons are glucose responsive and express K(ATP) channels. Endocrinology. 2003; 144(4):1331-40. doi: 10.12 10/en.2002-221033.

28. Blouet C, Jo YH, Li X, Schwartz GJ. Mediobasal hypothalamic leucine sensing regulates food intake through activation of a hypothalamus-brainstem circuit. J Neurosci. 2009; 29(26):8302-11. doi: 10.1523/JNEUROSCI.1668-09.2009.

29. Fioramonti X, Contie S, Song Z, Routh VH, Lorsignol A, Penicaud L. Characterization of glucosensing neuron subpopulations in the arcuate nucleus: integration in neuropeptide $Y$ and pro-opio melanocortin networks? Diabetes. 2007; 56(5): 1219-27. doi: 10.2337/db06-0567.

30. Jo YH, Su Y, Gutierrez-Juarez R, Chua S, Jr. Oleic acid directly regulates POMC neuron excitability in the hypothalamus. J Neurophysiol. 2009; 101(5): 2305-16. doi: 10.1152/jn.91294.2008.

31. Parton LE, Ye CP, Coppari R, Enriori PJ, Choi B, Zhang $C Y$, et al. Glucose sensing by POMC neurons regulates glucose homeostasis and is impaired in obesity. Nature. 2007; 449(7159):228-32. doi: 10.1038/nature06098.

32. Cha SH, Hu Z, Chohnan S, Lane MD. Inhibition of hypothalamic fatty acid synthase triggers rapid activation of fatty acid oxidation in skeletal muscle. Proc Natl Acad Sci USA. 2005; 102(41):14557-62. doi: 10.1073/pnas.0507300102.

33. Gao S, Lane MD. Effect of the anorectic fatty acid synthase inhibitor C75 on neuronal activity in the hypothalamus and brainstem. Proc Natl Acad Sci USA. 2003; 100(10):5628-33. doi: 10.1073/pnas. 1031698100.

34. Lucas A. Programming by early nutrition in man. Ciba Found Symp. 1991; 156:38-50; discussion 50-5.
35. Bateson $P$, Barker $D$, Clutton-Brock $T$, Deb $D$, D'Udine B, Foley RA, et al. Developmental plasticity and human health. Nature. 2004; 430(6998): 419-21. doi: 10.1038/nature02725.

36. Desai M, Gayle D, Babu J, Ross MG. Permanent reduction in heart and kidney organ growth in offspring of undernourished rat dams. Am J Obstet Gynecol. 2005; 193(3 Pt 2):1224-32. doi: 10.1016/ j.ajog.2005.05.041.

37. Toscano $A E$, Amorim MA, de Carvalho Filho EV, Aragao RS, Cabral-Filho JE, de Moraes SR, et al. Do malnutrition and fluoxetine neonatal treatment program alterations in heart morphology? Life Sci. 2008; 82(21-22):1131-6. doi: 10.1016/j.Ifs. 2008.03.013.

38. Toscano AE, Ferraz KM, Castro RM, Canon F. Passive stiffness of rat skeletal muscle undernourished during fetal development. Clinics. 2010; 65(12): 1363-9. doi: S1807-59322010001200022.

39. Costa-Cruz RR, Amancio-dos-Santos A, Guedes RC. Characterization of cortical spreading depression in adult well-nourished and malnourished rats submitted to the association of pilocarpine-induced epilepsy plus streptozotocin-induced hyperglycemia. Neurosci Lett. 2006; 401(3):271-5.

40. Do Monte-Silva KK, Assis FL, Leal GM, Guedes RC. Nutrition-dependent influence of peripheral electrical stimulation during brain development on cortical spreading depression in weaned rats. Nutr Neurosci. 2007; 10(3-4):187-94.

41. Vickers $M H$, Breier $B H$, Cutfield WS, Hofman PL, Gluckman PD. Fetal origins of hyperphagia, obesity, and hypertension and postnatal amplification by hypercaloric nutrition. Am J Physiol Endocrinol Metab. 2000; 279(1):E83-7.

42. Coupe B, Grit I, Darmaun D, Parnet P. The timing of "catch-up growth" affects metabolism and appetite regulation in male rats born with intrauterine growth restriction. Am J Physiol Regul Integr Comp Physiol. 2009; 297(3):R813-24. doi: 10.1152/ajpregu.00201.2009.

43. Lopes de Souza S, Orozco-Solis R, Grit I, Manhaes de Castro R, Bolanos-Jimenez F. Perinatal protein restriction reduces the inhibitory action of serotonin on food intake. Eur J Neurosci. 2008; 27(6):1400-8. doi: 10.1111/j.1460-9568.2008.06105.x.

44. Bayol S, Jones D, Goldspink G, Stickland NC. The influence of undernutrition during gestation on skeletal muscle cellularity and on the expression of genes that control muscle growth. Br J Nutr. 2004; 91(3):331-9. doi: 10.1079/BJN20031070.

45. Kensara OA, Wootton SA, Phillips DI, Patel M, Jackson AA, Elia M. Fetal programming of body composition: relation between birth weight and body composition measured with dual-energy X-ray absorptiometry and anthropometric methods 
in older Englishmen. Am J Clin Nutr. 2005; 82(5):980-7.

46. Fahey AJ, Brameld JM, Parr T, Buttery PJ. The effect of maternal undernutrition before muscle differentiation on the muscle fiber development of the newborn lamb. J Anim Sci. 2005; 83(11): 2564-71.

47. Brameld JM. The influence of undernutrition on skeletal muscle development. Br J Nutr. 2004; 91(3):327-8. doi: 10.1079/BJN20031077.

48. Mallinson JE, Sculley DV, Craigon J, Plant R, LangleyEvans SC, Brameld JM. Fetal exposure to a maternal low-protein diet during mid-gestation results in muscle-specific effects on fibre type composition in young rats. Br J Nutr. 2007; 98(2):292-9. doi: 10.1017/S0007114507701678.

49. Leandro CG, da Silva Ribeiro W, Dos Santos JA, Bento-Santos A, Lima-Coelho CH, Falcao-Tebas F, et al. Moderate physical training attenuates musclespecific effects on fibre type composition in adult rats submitted to a perinatal maternal low-protein diet. Eur J Nutr. 2011. doi: 10.1007/s00394-011-02 59-3.

50. McGarry JD. Banting lecture 2001: dysregulation of fatty acid metabolism in the etiology of type 2 diabetes. Diabetes. 2002; 51(1):7-18. doi: 10.2337/ diabetes.51.1.7.

51. Savage DB, Petersen KF, Shulman GI. Disordered lipid metabolism and the pathogenesis of insulin resistance. Physiol Rev. 2007; 87(2):507-20. doi: 10.1152/physrev.00024.2006.

52. van Loon LJ, Goodpaster BH. Increased intramuscular lipid storage in the insulin-resistant and endurance-trained state. Pflugers Arch. 2006; 451(5):606-16. doi: 10.1007/s00424-005-1509-0.

53. Rolo AP, Palmeira CM. Diabetes and mitochondrial function: role of hyperglycemia and oxidative stress. Toxicol Appl Pharmacol. 2006; 212(2):167-78. doi: 10.1016/j.taap.2006.01.003.

54. Camm EJ, Martin-Gronert MS, Wright NL, Hansell JA, Ozanne SE, Giussani DA. Prenatal hypoxia independent of undernutrition promotes molecular markers of insulin resistance in adult offspring. FASEB J. 2010; 25(1):420-7. doi: 10.1096/fj.10-15 8188.

55. Thompson NM, Norman AM, Donkin SS, Shankar RR, Vickers $M H$, Miles JL, et al. Prenatal and postnatal pathways to obesity: different underlying mechanisms, different metabolic outcomes. Endocrinology. 2007; 148(5):2345-54. doi: 10.12 10/en.2006-1641.

56. Morton GJ, Schwartz MW. The NPY/AgRP neuron and energy homeostasis. Int J Obes Relat Metab Disord. 2001; 25(Suppl 5):S56-62. doi: 10.1038/ sj.ijo.0801915.

57. Cha SH, Rodgers JT, Puigserver P, Chohnan S, Lane MD. Hypothalamic malonyl-CoA triggers mitochondrial biogenesis and oxidative gene expression in skeletal muscle: Role of PGC-1alpha. Proc Natl Acad Sci USA. 2006; 103(42):15410-5. doi: 10.1073/pnas.0607334103.

58. Broberger $C$. Brain regulation of food intake and appetite: molecules and networks. J Intern Med. 2005; 258(4):301-27. doi: 10.1111/j.1365-2796.2 005.01553.x.

59. Cechetto DF, Saper CB. Neurochemical organization of the hypothalamic projection to the spinal cord in the rat. J Comp Neurol. 1988; 272(4):579-604. doi: $10.1002 /$ cne. 902720410 .

60. Clapham JC, Arch JR, Chapman H, Haynes A, Lister $C$, Moore GB, et al. Mice overexpressing human uncoupling protein-3 in skeletal muscle are hyperphagic and lean. Nature. 2000; 406(6794): 415-8. doi: 10.1038/35019082.

61. Orozco-Solis R, Matos RJ, Guzman-Quevedo O, Lopes de Souza S, Bihouee A, Houlgatte R, et al. Nutritional programming in the rat is linked to longlasting changes in nutrient sensing and energy homeostasis in the hypothalamus. PLoS One. 2010; 5(10):e13537. doi: 10.1371/journal.pone.00135 37.

62. Young JB. Developmental origins of obesity: a sympathoadrenal perspective. Int J Obes (London). 2006; 30(Suppl 4):S41-9. doi: 10.1038/sj.ijo.08035 18.

63. Petry CJ, Dorling MW, Wang CL, Pawlak DB, Ozanne SE. Catecholamine levels and receptor expression in low protein rat offspring. Diabet Med. 2000; 17(12):848-53.

64. Fernandez-Twinn DS, Ekizoglou S, Wayman A, Petry CJ, Ozanne SE. Maternal low-protein diet programs cardiac beta-adrenergic response and signaling in 3-mo-old male offspring. Am J Physiol Regul Integr Comp Physiol. 2006; 291(2):R429-36. doi: 10.11 52/ajpregu.00608.2005.

65. Barker D, Saito M. Autonomic innervation of receptors and muscle fibres in cat skeletal muscle. Proc R Soc Lond B Biol Sci. 1981; 212(1188):317-32.

66. Navegantes LC, Baviera AM, Kettelhut IC. The inhibitory role of sympathetic nervous system in the Ca2+-dependent proteolysis of skeletal muscle. Braz J Med Biol Res. 2009; 42(1):21-8. doi: S010 0-879X2009000100005.

67. Eikelis N, Esler M. The neurobiology of human obesity. Exp Physiol. 2005; 90(5):673-82. doi: 10.11 13/expphysiol.2005.031385.

68. Hesselink MK, Mensink M, Schrauwen P. Human uncoupling protein-3 and obesity: an update. Obes Res. 2003; 11(12):1429-43. doi: 10.1038/oby.20 03.192.

Recebido em: 14/7/2011 Versão final em: $27 / 3 / 2012$ Aprovado em: 17/4/2012 
